\title{
COMPARISON OF THREE MULTICRITERIA DECISION MAKING THEORIES: The Analytic Hierarchy Process, Multiattribute Utility Theory and OutRanking Methods
}

\author{
Luis G. Vargas \\ University of Pittsburgh
}

\section{Introduction}

There are three clearly distinct theories of decision making in practice today: The Analytic Hierarchy Process (AHP), Multiattribute Utility Theory (MAUT) and OutRanking Methods (ORM). Their main differences reside with the implementation scale and the philosophical principles on which they are built. The AHP uses ratio scales and assumes that the rank preservation principle does not hold. MAUT is built on interval scales and upholds the principle of rank preservation. ORM uses ordinal scales and does not assume anything about rank preservation. It is built on the principle of dominating alternatives. ORM is the preferred method by many researchers of the French School and MAUT is predominantly used by English speaking researchers, the American and British Schools. The AHP is used all over the world.

The objective of this paper is to present the three theories side by side in the framework of measurement theory to show their differences and similarities. We will use the same example with the three theories to help explain how they differ in their implementation. In the process of developing the example we will attempt to obtain the same result by all the theories to better understand how they work and the type of information that is required to obtain an answer. We start by introducing the primitives of these theories.

\section{Primitives}

Primitives are the elements of a theory on which the axioms are based. Let $C$ be a set of properties or attributes. We usually refer to them as criteria; let $\mathcal{A}$ be a set of n alternatives; let $O$ be the space of outcomes or consequences from the alternatives; and let $L$ be the set of lotteries built on the space of outcomes $O, O \subset \mathcal{L}$. Let $\geq_{C}$ be a binary relation representing "more preferred than or indifferent to" according to $C \in C$. Let

$$
\begin{gathered}
\mathcal{U} \equiv\{\mathrm{u} \mid \mathrm{u}: \mathcal{L} \rightarrow \mathscr{R}\}, \\
\mathcal{W} \equiv\{\mathrm{w} \mid \mathrm{w}: \mathcal{A} \rightarrow[0,1]\}, \\
\Omega \equiv\{\varphi \mid \varphi: \mathcal{A} \times \mathcal{A} \rightarrow \mathscr{R}\}, \\
\Theta \equiv\{\psi \mid \psi: \mathcal{L} \times \mathcal{L} \rightarrow \mathscr{R}\} .
\end{gathered}
$$

Let $\pi: C \rightarrow \Omega$ and $\phi: C \rightarrow \Theta$ be mappings from the set of criteria to the set of pairwise comparison functions. Thus for all $C \in C$ there exists a $\pi(\varphi)=\varphi_{C} \in \Omega$ and a $\phi(\psi)=\psi_{C} \in \Theta$ such that

$$
\begin{aligned}
& A_{i}>{ }_{C} A_{j} \text { if and only if } \varphi_{C}\left(A_{i}, A_{j}\right)>1 \\
& A_{i} \sim{ }_{C} A_{j} \text { if and only if } \varphi_{C}\left(A_{i}, A_{j}\right)=1
\end{aligned}
$$


or

$$
\begin{aligned}
& A_{i}>_{C} A_{j} \text { if and only if } \psi_{C}\left(A_{i}, A_{j}\right)>0 \\
& A_{i} \sim_{C} A_{j} \text { if and only if } \psi_{C}\left(A_{i}, A_{j}\right)=0 .
\end{aligned}
$$

In both theories, the AHP and Utility Theory, the problem is the same:

Find a function $u \in \mathcal{U}$ (or $w \in \mathcal{W})$ which captures the preferences; of the decision maker through the use of $\psi_{C}\left(\right.$ or $\left.\varphi_{C}\right)$.

Although the problem is almost identical, the assumptions are quite different:

- In UT one deals with the outcomes of the actions, and hence the probability distributions of the outcomes play a significant role in the evaluation of the alternatives. In the AHP, the likelihood of occurrence of the outcomes may change from attribute to attribute and thus, we deal with the alternatives rather than with the probability distribution of their outcomes.

- UT does not use pairwise comparisons to construct the utility function. Instead, the utility function $\mathrm{u}(\mathrm{x})$ is built directly using some of the available methods and $\psi(\mathrm{x}, \mathrm{y})=\mathrm{u}(\mathrm{x})-\mathrm{u}(\mathrm{y})$. The AHP is built on the concept of pairwise comparison and does not make any assumptions on the type of order implied by the paired comparisons. Instead, emphasis is put on the strength of preferences and their inconsistency.

\section{The Single Attribute Case}

\section{Utility Theory}

(U1): $>_{C}$ on $\mathcal{L}$ is a weak order.

(U2): $\forall \mathrm{x}, \mathrm{y}, \mathrm{z} \in \mathcal{L}$,

if $\mathrm{x}>_{C} \mathrm{y}$ and $0<\lambda<1$

then $\lambda \mathrm{x}+(1-\lambda) \mathrm{z}>_{C} \lambda \mathrm{y}+(1-\lambda) \mathrm{z}$

(U3): $\forall \mathrm{x}, \mathrm{y}, \mathrm{z} \in \mathcal{L}$, if $\mathrm{x}>_{c} \mathrm{y}$ and

$\mathrm{y}>_{C} \mathrm{z}$, then $\exists \alpha, \beta, 0<\alpha, \beta<1$

for which

$\alpha x+(1-\alpha) z>_{C} y>_{C} \beta y+(1-\beta) z$
Axioms

of

Analytic Hierarchy Process

(H1): (Reciprocity)

$\forall \mathrm{A}_{\mathrm{i}} \mathrm{A}_{\mathrm{j}} \in \mathcal{A} \varphi_{\mathrm{C}}\left(\mathrm{A}_{\mathrm{i}}, \mathrm{A}_{\mathrm{j}}\right) \varphi_{\mathrm{C}}\left(\mathrm{A}_{\mathrm{j}}, \mathrm{A}_{\mathrm{i}}\right)=1$

(H2): (Boundedness)

$\exists \rho>0$ for which $\frac{1}{\rho}<\varphi_{C}\left(A_{i}, A_{j}\right)<\rho$.

The result of the representation theorem of the AHP is a ratio scale given by the principal eigenvector of the reciprocal matrix whose entries are the paired comparisons defined by $\varphi_{C}$. 


\section{Representation and Uniqueness}

The axioms U1-U3 hold if and only if there is a real value function $u \in U$ on $L$ that is:

and

(1) Order-preserving:

$\forall \mathrm{x}, \mathrm{y} \in \mathcal{L} \mathrm{x}>_{C} \mathrm{y}$ iff $\mathrm{u}(\mathrm{x})>\mathrm{u}(\mathrm{y})$,

(2) Linear:

$\forall \mathrm{x}, \mathrm{y} \in \mathcal{L}, \forall \lambda \in[0,1]$,

$u[\lambda x+(1-\lambda) y]=\lambda u(x)+(1-\lambda) u(y)$
I The axioms H1-H2 hold if and only if there is a | real value function $w \in W$ on $A$ that is:

(1) Order-preserving

$\mathrm{A}_{\mathrm{i}}>{ }_{C} \mathrm{~A}_{\mathrm{j}}$ iff $\exists \mathrm{N}: \forall \mathrm{m}>\mathrm{N}$

$\frac{1}{m} \sum_{k=1}^{m} \sum_{h=1}^{n} a_{i h}^{(k)}>\frac{1}{m} \sum_{k=1}^{m} \sum_{h=1}^{n} a_{j h}^{(k)}$

where $a_{i h}^{(k)} \equiv(\mathrm{i}, \mathrm{h})$ entry of the matrix $\mathrm{A}^{\mathrm{k}}$,

$\mathrm{A}=\left(a_{i h}\right), a_{i h}=\varphi_{C}\left(A_{i}, A_{h}\right)$, and

$w=\lim _{m \rightarrow \infty} \frac{1}{m} \sum_{k=1}^{m} \sum_{h=1}^{n} a_{i h}^{(k)}$

I and

(3) Unique up to a positive affine |
transformation

(2) Unique up to a similarity transformation

\section{The Multiattribute Case}

There are two possibilities when dealing with multiattributes. Either the axiom of transitivity is satisfied for all the criteria, as it is in Multiattribute Linear Utility Theory (Keeney and Raiffa, 1976), or it is not satisfied as in the Analytic Hierarchy Process (Saaty, 1993) and Multiattribute Nonlinear Utility Theory (Fishburn, 1984).

\section{Multiattribute Linear Utility Theory}

An axiom of independence is added to the axioms U1-U3:

(U4): All attributes are mutually utility independent.

An attribute $\mathrm{C}_{\mathrm{j}} \in \mathrm{C}$ is utility independent (U.I.) of another attribute $\mathrm{C}_{\mathrm{j}} \in C$ if, and only if the conditional preferences for lotteries on $C_{i}$ given a level of $C_{j}$ do not depend on the particular level of $C_{j}$. If $C_{i}$ is U.I. of $C_{j}$, and $C_{j}$ is U.I. of $C_{i}$, then they are said to be mutually utility independent (M.U.I.).

The representation theorem states:

The axioms U1-U4 hold if, and only if there is a real value function $u \in U$ on $L$ given by:

$u\left(x_{1}, \ldots, x_{n}\right)=\sum_{i=1}^{n} k_{i} u_{i}\left(x_{i}\right)+\sum_{i=1}^{n} \sum_{j>i} k_{i j} u_{i}\left(x_{i}\right) u_{j}\left(x_{j}\right)+\sum_{i=1}^{n} \sum_{j>i} \sum_{s>j} k_{i j s} u_{i}\left(x_{i}\right) u_{j}\left(x_{j}\right) u_{s}\left(x_{s}\right)+\cdots+k_{123 \ldots n} \prod_{i=1}^{n} u_{i}\left(x_{i}\right)$ 


\section{Multiattribute Nonlinear Utility Theory}

To introduce this theory we need to assume that the alternatives can be represented by probability distributions on the space of outcomes $O$. Thus, $\mathrm{x}$ now denotes the probability distribution of the outcomes. For every criterion $C \in C$, the axioms of the theory are as follows:

(F1): (Continuity)

If $\mathrm{x}>_{C} \mathrm{y}$ and $\mathrm{y}>_{c} \mathrm{z}$ then $\exists \lambda, 0<\lambda<1$, for which $\mathrm{y} \sim_{c} \lambda \mathrm{x}+(1-\lambda) \mathrm{y}$

(F2): (Dominance)

If $\mathrm{x}>_{C} \mathrm{y}$ and $\mathrm{x}>_{c} \mathrm{z}$ then $\mathrm{x}>_{c} \lambda \mathrm{y}+(1-\lambda) \mathrm{z}$, for all $\lambda, 0<\lambda<1$.

If $y>_{C} x$ and $z>_{C} x$ then $\lambda y+(1-\lambda) z>_{C} x$, for all $\lambda, 0<\lambda<1$.

If $\mathrm{x} \sim{ }_{c} \mathrm{y}$ and $\mathrm{x} \sim{ }_{c} \mathrm{z}$ then $\mathrm{x} \sim{ }_{c} \lambda \mathrm{y}+(1-\lambda) \mathrm{z}$, for all $\lambda, 0<\lambda<1$.

(F3): (Symmetry)

If $\mathrm{x}>_{C} \mathrm{y}, \mathrm{y}>_{c} \mathrm{z}, \mathrm{x}>_{C} \mathrm{z}$ and $\mathrm{y} \sim_{c} \frac{1}{2} x+\frac{1}{2} z$ then $\exists \lambda, 0<\lambda<1$, for

which $\lambda x+(1-\lambda) z \sim c \frac{1}{2} x+\frac{1}{2} y$.

(F4): (Marginal Indifference)

$\forall \mathrm{x}, \mathrm{y} \in \mathcal{L}$, if $\mathrm{x}$ and $\mathrm{y}$ have the same marginal distributions, then they are indifferent according to all the attributes.

(F5): (Marginal Preference)

$\forall \mathrm{x}, \mathrm{y}, \mathrm{z}, \mathrm{v} \in \mathcal{L}$, if $\mathrm{x}$ has marginals $\left(\mathrm{x}_{1}, \mathrm{x}_{2}, \ldots, \mathrm{x}_{\mathrm{n}}\right)$,

$y$ has marginals $\left(x_{1}, y_{2}, \ldots, x_{n}\right)$,

$z$ has marginals $\left(z_{1}, x_{2}, \ldots, x_{n}\right)$,

and

$v$ has marginals $\left(z_{1}, y_{2}, \ldots, x_{n}\right)$,

then $x>z$ implies $y>v$, and $x>y$ implies $z>v$.

The representation theorem states:

The axioms F1-F3 hold if, and only if there is a skew-symmetric bilinear functional $\psi$ on $\mathcal{L} \times \mathcal{L}$ such that:

(1) For all $\mathrm{x}, \mathrm{y} \in \mathcal{L}, \mathrm{x}>\mathrm{y}$ if and only if $\psi(\mathrm{x}, \mathrm{y})>0$,

(2) It is unique up to a similarity transformation.

Fishburn (1988) also provides the form of the functional $\psi$ if $\mathbf{F} 4$ holds, and if F4 does not hold but $\mathbf{F 5}$ does. However, it is difficult to establish a relationship between the individual utilities $\mathrm{u}_{\mathrm{i}}\left(\mathrm{x}_{\mathrm{i}}\right)$ and $\psi(\mathrm{x}, \mathrm{y})$. 


\section{The Analytic Hierarchy Process}

To deal with multiple attributes in the AHP two concepts of dependence are needed. The set of alternatives $\mathcal{A}$ is said to be outer dependent (independent) on a criterion $\mathrm{C}$ if there (does not) exists a $\mathrm{w}_{C} \in \mathcal{W}$ associated with them. The set of alternatives $\mathcal{A}$ is said to be inner dependent with respect to a criterion $\mathrm{C}$ if, and only if the elements in $\mathrm{A}$ are outer dependent on themselves according to the criterion.

\section{(H3): (Independence)}

In a hierarchy

(i) A level is outer dependent on the level above it,

(ii) A level is inner independent with respect to all

the elements in the level above it,

(iii) A level is outer independent on the level below it.

(H4): (Expectations)

When making a decision the hierarchy is assumed complete.

Let $H_{m}$ be a hierarchy with $m$ levels. Let $w\left(L_{i+1}\left[L_{i}\right)\right.$ be the scales derived for the elements in the $(i+1)$ st level with respect to the elements in the ith level. $w\left(L_{i+1} \mid L_{i}\right)$ is a matrix operator with the number of rows and columns equal to the number of elements in $\mathrm{L}_{\mathrm{i}+1}$ and $\mathrm{L}_{\mathrm{i}}$, respectively.

The representation theorem states:

The axioms H1-H4 hold if, and only if the scale associated with a level $\mathrm{L}_{k}$ is given by:

$$
\mathrm{w}\left(\mathrm{L}_{\mathrm{k}} \mid \mathrm{L}_{1}\right)=\mathrm{w}\left(\mathrm{L}_{\mathrm{k}} \mid \mathrm{L}_{\mathrm{k}-1}\right) \mathrm{w}\left(\mathrm{L}_{\mathrm{k}-1} \mid \mathrm{L}_{\mathrm{k}-2}\right) \cdots \mathrm{w}\left(\mathrm{L}_{2} \mid \mathrm{L}_{\mathrm{l}}\right) \mathrm{w}\left(\mathrm{L}_{1}\right) .
$$

\section{- Outranking Methods:}

\section{Electre I}

The first of these methods (Electre I) was developed by Bernard Roy (1968). He introduced the concept of an outranking relation $S$ as a binary relation defined on the set of alternatives $\mathcal{A}$. Given two alternatives $A_{j}$ and $A_{j}, A_{i}$ outranks $A_{j}$, or $A_{j} S A_{j}$, if given all that it is known about the two alternatives, there are enough arguments to decide that $A_{i}$ is at least as good as $A_{j}$. The goal of outranking methods is to find all alternatives that dominate other alternatives while they cannot be dominated by any other alternative. To find the best alternative the criteria weights are assumed to be measured on some scale, probably a ratio scale. Each criterion $C_{\mathrm{j}} \in C$ is assigned a weight $w_{j}$, and every pair of alternatives $A_{i}$ and $A_{j}$ is assigned a concordance index $c\left(A_{i}, A_{j}\right)$ given by:

$$
c\left(A_{i}, A_{j}\right)=\frac{1}{\sum_{k=1}^{n} w_{k}} \sum_{\left.k: \mathbb{B}_{k}\left(A_{i}\right) \geq \mathfrak{B}_{k}\left(A_{j}\right)\right\}} w_{k}
$$

and a discordance index $d\left(A_{i}, A_{j}\right)$ given by: 


$$
d\left(A_{i}, A_{j}\right)=\left\{\begin{array}{l}
0 \text { if } g_{k}\left(A_{i}\right) \geq g_{k}\left(A_{j}\right) \text { for all } k, \\
\frac{1}{\delta} \max _{k}\left\{g_{k}\left(A_{j}\right)-g_{k}\left(A_{i}\right)\right\}, \text { otherwise. }
\end{array}\right.
$$

where $\delta=\max _{k, A_{\mathrm{i}}, A_{\mathrm{j}}}\left\{\mathrm{g}_{\mathrm{k}}\left(\mathrm{A}_{\mathrm{i}}\right)-\mathrm{g}_{\mathrm{k}}\left(\mathrm{A}_{\mathrm{j}}\right)\right\}$. Obviously, the discordance index is only valid if the operation subtraction is well defined. Once the two indices are defined, an outranking relation $S$ is defined by :

$$
\mathrm{A}_{\mathrm{i}} \mathrm{SA}_{\mathrm{j}} \text { if and only if }\left\{\begin{array}{l}
c\left(A_{i}, A_{j}\right) \geq \hat{c}, \\
d\left(A_{i}, A_{j}\right) \leq \hat{d},
\end{array}\right.
$$

where $\hat{c}$ and $\hat{d}$ are thresholds. A problem with this discordance index is that the criteria levels be quantifiable. If that is not the case, then a discordance set $D_{j}$ is defined for each criterion $j$ with all the ordered pairs $\left(x_{j}, y_{j}\right)$ such that if $g_{j}(A)=x_{j}$ and $g_{j}(B)=y_{j}$ then the outranking of $B$ by $\Lambda$ is refused. The outranking relation is defined now:

$$
A_{i} S A_{j} \text { if and only if }\left\{\begin{array}{c}
c\left(A_{i}, A_{j}\right) \geq \hat{c}, \\
\left(g_{j}\left(A_{i}\right), g_{j}\left(A_{j}\right)\right) \notin D_{j}, \forall j .
\end{array}\right.
$$

Given the outranking relation it is now possible to find the set of alternatives $\mathcal{N} \subseteq \mathcal{A}$ for which:

$$
\begin{gathered}
\forall \mathrm{B} \in \mathcal{A}-\mathcal{N}, \exists \mathrm{A} \in \mathcal{N} \text { such that } \mathrm{ASB} \\
\forall \mathrm{A} \in \mathcal{N}, \mathrm{A} \$ \mathrm{~B} .
\end{gathered}
$$

The outranking relation determines the set of non-dominated alternatives. The alternatives in $\mathcal{N}$ form the kernel of the graph defined by the alternatives (vertices) and the outranking relation (edges). Thus, if alternative $A_{i}$ outranks alternative $A_{j}$, then a directed arc exists from $A_{i}$ to $A_{j}:$ : $A_{i} \rightarrow A_{j}$.

There are three other variations of this method depending on how the outranking relation is defined. The method most employed in applications requiring ranking of the alternatives rather than choice is based on an outranking relation in which the concordance and the discordance indices have two levels used to define a strong and a weak outranking relation. This method is known as Electre II [Roy and Bertier, 1973].

\section{An Example}

A group of researchers in the process of solving a problem develop software which can be used in a variety of forms to accomplish objectives such as (1) do research and obtain funds to buy the researchers time (RESEARCH), (2) develop a product and market it (MKTDEV), (3) capture some share of the market in the industry in question (MKTSHARE), and (4) make money (PROFIT). These objectives can be attained following different courses of action: (a) independent commercialization of the product (INDCOMM), (b) form a joint venture with a company that has pursued similar projects in the past (JOINTVEN), (c) relinquish the right of the product to the institution where they are affiliated and collect royalties (NORIGHTS), and (d) obtain funding from independent sources and use them to do research and consulting with the tool developed 
(INDFUNDS). The matrices of paired comparisons and the corresponding priorities are given in Table 1.

The decision the AHP model suggests is to obtain independent funding and use it to do $\mathrm{R} \& \mathrm{D}(0.389)$. A close second alternative is to pursue a joint venture with a company that has done this type of work in the past (0.335).

Table 1. Pairwise comparisons and Priorities from the AHP model

\begin{tabular}{|llllll|}
\hline Best Option & 1 & 2 & 3 & 4 & Priorities \\
1.RESEARCH & 1 & 3 & 3 & 3 & 0.479 \\
2.MKTDEV & & 1 & 1 & 3 & 0.199 \\
3.MKTSHARE & & & 1 & 5 & 0.238 \\
4. PROFIT & & & & 1 & 0.084 \\
& & & & & $\mathrm{CR}=0.099$ \\
\hline
\end{tabular}

\begin{tabular}{|cccccc|}
\hline RESEARCH & $\mathrm{a}$ & $\mathrm{b}$ & $\mathrm{c}$ & $\mathrm{d}$ & Priorities \\
$\mathrm{a}$ & 1 & $1 / 5$ & 3 & $1 / 7$ & 0.082 \\
$\mathrm{~b}$ & & 1 & 5 & $1 / 5$ & 0.230 \\
$\mathrm{c}$ & & & 1 & $1 / 9$ & 0.044 \\
$\mathrm{~d}$ & & & & 1 & 0.644 \\
& & & & & $\mathrm{CR}=0.111$ \\
\hline MKTDEV & $\mathrm{a}$ & $\mathrm{b}$ & $\mathrm{c}$ & $\mathrm{d}$ & Priorities \\
$\mathrm{a}$ & 1 & 3 & 7 & 5 & 0.574 \\
$\mathrm{~b}$ & & 1 & 5 & 2 & 0.239 \\
$\mathrm{c}$ & & & 1 & $1 / 3$ & 0.056 \\
$\mathrm{~d}$ & & & & 1 & 0.131 \\
& & & & & $\mathrm{CR}=0.029$ \\
\hline
\end{tabular}

\begin{tabular}{|cccccc|}
\hline MKTSHARE & $\mathrm{a}$ & $\mathrm{b}$ & $\mathrm{c}$ & $\mathrm{d}$ & Priorities \\
$\mathrm{a}$ & 1 & $1 / 5$ & 1 & 1 & 0.112 \\
$\mathrm{~b}$ & & 1 & 7 & 7 & 0.666 \\
$\mathrm{c}$ & & & 1 & $1 / 3$ & 0.080 \\
$\mathrm{~d}$ & & & & 1 & 0.141 \\
& & & & & $\mathrm{CR}=0.063$ \\
\hline
\end{tabular}

Table 1. (Cont.)

\begin{tabular}{|cccccc|}
\hline PROFIT & $\mathrm{a}$ & $\mathrm{b}$ & $\mathrm{c}$ & $\mathrm{d}$ & Priorities \\
$\mathrm{a}$ & 1 & 3 & 2 & 2 & 0.470 \\
$\mathrm{~b}$ & & 1 & 5 & 1 & 0.225 \\
$\mathrm{c}$ & & & 1 & $1 / 5$ & 0.060 \\
$\mathrm{~d}$ & & & & 1 & 0.244 \\
& & & & & $\mathrm{CR}=0.044$ \\
\hline
\end{tabular}

\begin{tabular}{|c|c|}
\hline Actions & $\begin{array}{c}\text { Composite } \\
\text { Priorities }\end{array}$ \\
\hline a.INDCOMM & 0.220 \\
b.JOINTVEN & 0.335 \\
c.NORIGHTS & 0.056 \\
d.INDFUNDS & 0.389 \\
\cline { 2 - 2 } & $\mathrm{CR}=0.09$ \\
\hline
\end{tabular}


We now use these priorities to construct utility functions for each criterion under the assumption that an additive multiattribute utility model is applicable. Table 2 summarizes these results.

The utilities of the alternatives for each criterion are obtained by subtracting the priority of the lowest alternative from all the priorities and dividing by the largest resulting priority. By definition, the least preferred alternative receives a utility of zero and the most preferred alternative a utility of one. In the example, we assumed that the scaling constants $k_{i}, i=1,2, \ldots, n$ are equal to the weights of the criteria. The multiattribute utility function is given by

$$
u\left(x_{1}, x_{2}, \ldots, x_{n}\right)=\sum_{i=1}^{n} k_{i} u_{i}\left(x_{i}\right)+P O T
$$

where POT stands for possible other terms. The composite utility of each alternative is obtained by using an additive model given by:

$$
u\left(x_{1}, x_{2}, \ldots, x_{n}\right)=\sum_{i=1}^{n} k_{i} u_{i}\left(x_{i}\right)
$$

if $\sum_{i=1}^{n} k_{i}=1$. If on the other hand, an additive model is not applicable because the attributes are shown to be mutually utility independent, then a multiplicative model must be used:

$$
u\left(x_{1}, x_{2}, \ldots, x_{n}\right)=-\frac{1}{k}+\frac{1}{k} \prod_{i=1}^{n}\left[k k_{i} u_{i}\left(x_{i}\right)+1\right]
$$

If the attributes are utility independent of their complement, i.e., all other attributes are held at their most preferred level and the attribute in question is held at its least preferred level, then the utility function is multilinear and it is given by:

$$
\begin{aligned}
& u\left(x_{1}, x_{2}, \ldots, x_{n}\right)= \\
& \quad \sum_{i=1}^{n} k_{i} u_{i}\left(x_{i}\right)+\sum_{i=1}^{n} \sum_{j>i} k_{i j} u_{i}\left(x_{i}\right) u_{j}\left(x_{j}\right)+\sum_{i=1}^{n} \sum_{j>i} \sum_{i>j} k_{i j l} u_{i}\left(x_{i}\right) u_{j}\left(x_{j}\right) u_{l}\left(x_{l}\right)+\cdots+k_{12 \ldots n} \prod_{i=1}^{n} u_{i}\left(x_{i}\right) .
\end{aligned}
$$

Table 2. Utility functions and Utilities of the Alternatives

\begin{tabular}{cccccc|}
\hline $\begin{array}{c}\text { Criteria } \rightarrow \\
\text { Alternatives }\end{array}$ & $\begin{array}{c}\text { RESEARCH } \\
(0.479)\end{array}$ & $\begin{array}{c}\text { MKTDEV } \\
(0.199)\end{array}$ & $\begin{array}{c}\text { MKTSHARE } \\
(0.238)\end{array}$ & $\begin{array}{c}\text { PROFIT } \\
(0.084)\end{array}$ & $\begin{array}{c}\text { Composite } \\
\text { Utility }\end{array}$ \\
$\begin{array}{ccccc}\text { INDCOMM } \\
\text { JOINTVEN }\end{array}$ & 0.063 & 1 & 0.055 & 1 & \\
NORIGHTS & 0.31 & 0.353 & 1 & 0.402 & 0.326 \\
INDFUNDS & 0 & 0 & 0 & 0 & 0.491 \\
\end{tabular}

The outranking method Electre I uses the outranking relation concept based on two indices, the concordance index and the discordance index. The former could be constructed using, for example, the priorities obtained in the AHP. For example, the concordance index of the 
alternatives $\mathrm{a}$ and $\mathrm{b}, \mathrm{C}(\mathrm{a}, \mathrm{b})$, is obtained by summing the weights of the criteria for which alternative a dominates alternative $b$. We have

$$
\begin{aligned}
& C(a, b)=0.199+0.084=0.283 \\
& C(b, a)=0.479+0.238=0.717
\end{aligned}
$$

The resulting index is given in Table 3.

Table 3. The Concordance Index

\begin{tabular}{|c|c|c|c|c|}
\hline & & & & \\
\hline $\mathrm{C}(\mathrm{x}, \mathrm{y})$ & $\mathrm{a}$ & $\mathrm{b}$ & $\mathrm{c}$ & $\mathrm{d}$ \\
\hline & & & & \\
\hline $\mathrm{b}$ & 0.717 & - & 1 & 0.238 \\
\hline $\mathrm{c}$ & 0.00 & 0 & - & 0 \\
\hline $\mathrm{d}$ & 0.717 & 0.762 & 1.00 & - \\
\hline
\end{tabular}

Table 4 summarizes this index for all the pairs of alternatives.

Table 4. The Discordance Index

\begin{tabular}{|c|c|c|c|c|}
\hline $\mathrm{d}(\mathrm{x}, \mathrm{y})$ & $\mathrm{a}$ & $\mathrm{b}$ & $\mathrm{c}$ & $\mathrm{d}$ \\
\hline & & & & \\
$\mathrm{a}$ & - & 0.558 & 0 & 0.738 \\
\hline & & & & \\
$\mathrm{b}$ & 0.923 & - & 0 & 0.875 \\
\hline $\mathrm{c}$ & 0.863 & 0.977 & - & 1.00 \\
\hline & & & & \\
\hline $\mathrm{d}$ & 0.937 & 0.69 & 0 & - \\
\hline
\end{tabular}


These two indices are now used to construct the outranking relation. First, for an alternative to outrank another, we must select the thresholds $\hat{c}$ and $\hat{d}$ above and below which the concordance and the discordance indices, respectively, must fall. For example, if $\hat{c}=0.25$ and $\hat{d}=.5$ then we obtain the graph given in Figure 1. Here the alternatives $\mathrm{a}, \mathrm{b}$ and $\mathrm{d}$ outrank $\mathrm{c}$ but nothing can be said about whether or not one prefers one alternative over another.

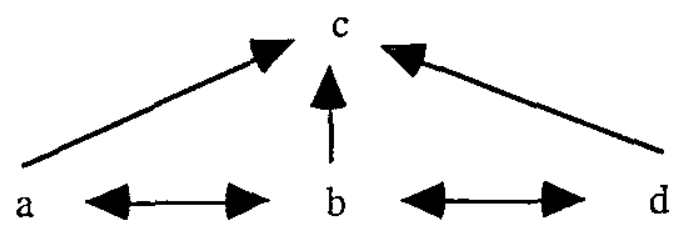

Figure 1

Making the discordance threshold $\hat{d}=0.7$ we obtain the graph given in Figure 2. Here it is possible to conclude that alternative $d$ dominates the others.

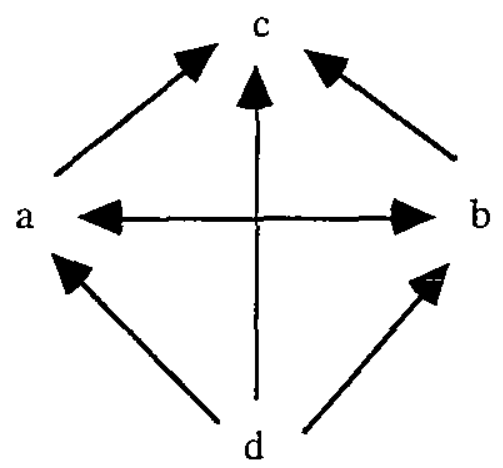

Figure 2

\section{Conclusions}

There are major philosophical and methodological differences between the three theories described above. The AHP produces ratio scales, MAUT yields interval scales and ORM (Electre I) derives ordinal scales. In the process of constructing the scales all three methods need quantification of criteria. A basic difference between the AHP and MAUT beyond the type of scales is that the criteria weights that appear in the multiattribute utility function do not represent how important the criteria weights are but speak of scaling constants (see Keeney and Raiffa, 1976). Many practitioners of MAUT do not seem to understand this difference. This characteristic of MAUT of not assigning importance weights to the criteria creates difficulties for decision makers when criteria ranges are varied (see Nitzsch and Weber, 1993). A problem that appears more insurmountable for MAUT is that in many real life situations the criteria are not easily quantifiable as required, nor are the alternatives easily measured under the criteria if there are no scales. This is also a problem with ORM (Electre I) although less damaging than for MAUT because only qualitative criteria levels are required to construct the concordance and discordance indices. Nonetheless, it still needs criteria weights to compute them. Later versions of Electre 
elaborate more on the evaluation of the alternatives under each criterion to refine the outranking relation. This would also make Electre more vulnerable to the same criticisms as MAUT, i.e., the criteria must be quantifiable for the alternatives to be evaluated.

In Electre a major issue is the thresholds used to define the outranking relation. They are selected by the decision maker. The concordance threshold defines the minimum amount by which an alternative must dominate another, and the discordance threshold sets the maximum amount by which an alternative can be dominated by another when it does not dominate it on all criteria. There does not appear to be any guidelines to set these thresholds. Because their values belong to the same scale as the scale of the criteria weights, their justification would be hard if, as MAUT followers assume, criteria weights cannot be estimated. The best one can do is to determine the marginal rate of substitution among units of different criteria.

A final methodological difference among the methods is the use of elaborate hierarchic and network structures to faithfully represent a decision problem and subsequently to apply a principle of composition to choose among the alternatives. In situations where no criteria scales are available, MAUT practitioners are hard pressed to find some way of quantifying the criteria to construct the utility functions and then compose them. Because the principle of composition used depends on,the subjective concept of independence defined in the theory, one can easly show that two different composition methods, e.g., the additive model or the multiplicative model, may not yield the same ranking of the alternatives. In those situations it is quite difficult to decide which solution is the appropriate one because expert users of MAUT can lead the decision maker to either one of the models just as easily. In ORM (Electre I) composition is assumed as part of the process. An alternative scores the full value of a criterion if it dominates another alternative on that criterion. The score of the alternative is obtained by adding all the criteria on which it dominates another alternative. In this step, it is assumed that all the criteria are equally weighted. The criticism mentioned above of MAUT could also be applied to the AHP if one thinks of hierarchic composition and the supermatrix. This indicates that composition principles are just as subjective as the concept of independence on which they are based. The basic distinction with the AHP is that from the start, it assumes that all is relative and subjective. Neither MAUT nor ORM (Electre) can use more than a two level structure to deal with complex decision problems. The AHP can because it composes ratio scales.

The major philosophical difference between the AHP and the other methods is that rank reversal is acceptable and that the criteria are just as measurable as alternatives are. Alternatives are assumed to be concrete instances of higher order concepts (e.g., criteria) which in turn are instantiations of higher order concepts (e.g., goals), and so on. MAUT stands at the opposite side of the spectrum. Criteria cannot be compared and rank reversal cannot take place. It is based on the belief that the only way to measure risk is through probabilities, and these probabilities are the ones that must be used to represent decision makers' preferences.

\section{References}

Keeney, R.L. and H. Raiffa, Decisions with Multiple Objectives: Preferences and Value Tradeoffs, Wiley, New York, 1976.

Fishburn, P., "SSB Utility Theory and Economic Perspectives," Math. Soc. Science 8 (1984) 63-94.

von Nitzsch, Rüdiger and Martin Weber, "The Effect of Attribute Ranges on Weights in Multiattribute Utility Measurements," Management Science 39, 8 (1993) 937-943.

Roy, B., "Classement et Choix en Présence de Points de Vue Multiples (la Méthode Electre)," Revue Française d'Informatique et de Recherche Opérationalle 8 (1968) 57-75.

Roy, B. and P. Bertier, La Méthode ELECTRE II, une Application au Média-planning," In $O R 72$, M. Ross (ed), North-Holland, 291-302, 1973.

Saaty, T.L., Multicriteria Decision Making: The Analytic Hierarchy Process, RWS Publications, 1993. 